\section{Getting under the skin of West Highland White Terriers using primary practice clinical data}

\section{Zoie F. Ballantyne, Dave C. Brodbelt, David B. Church, Dan G. O'Neill}

Royal Veterinary College, Hertfordshire, United Kingdom

\section{OBJECTIVES}

West Highland White Terriers (WHWT) are often considered by veterinarians as 'the itchy/scratchy breed' but little prevalence exists on their overall health. This study aimed to report demography, mortality and common disorders in WHWTs under UK primary veterinary care and to explore co-morbid disorder associations with otitis externa (OE).

\section{METHODS}

Demographic, mortality and disorder data for the year 2016 were extracted from VetCompass ${ }^{T M}$ de-identified patient records.

\section{RESULTS}

This study included 2053 WHWTs. Median age was 7.8 years (IQR 4.3-11.1). Of 164 deaths recorded, the median longevity was 13.4 years (IQR 11.0-15.0). The most commonly recorded causes of death were lower respiratory tract disorder $(10.2 \%, 95 \% \mathrm{Cl} 4.9-15.4)$, neoplasia $(10.2 \%$, $95 \% \mathrm{Cl} 4.9-15.4)$ and spinal cord disorder $7.8 \%(95 \% \mathrm{Cl}$ 3.2-12.5). The most common specific disorders were periodontal disease $(15.7 \%, 95 \% \mathrm{Cl} 14.1-17.3)$, OE $(10.6 \%$, 95\% Cl 9.3-11.9), overgrown nails (7.2\%, 95\% Cl 6.1-8.4), hypersensitivity (allergic) skin disorder $(6.5 \%, 95 \% \mathrm{Cl} 5.4-$ $7.6 \%)$ and obesity $(6.1 \%, 95 \% \mathrm{Cl} 5.1-7.2 \%)$. Of the top 20 disorders, no non-epidermal disorders were co-morbidly associated with OE while 3 of the 10 epidermal disorders (pododermatitis, pyoderma and dermatitis) were associated with $\mathrm{OE}$.

\section{STATEMENT (CONCLUSIONS)}

Compared with other common breeds, WHWTs were heavily over-represented for skin disease. High comorbidity between skin diseases suggests common underlying aetiopathogenesis pathways that may offer opportunities to control several skin conditions simultaneously. High mortality due to lower respiratory tract disease suggests that clinical focus should be placed on respiratory function in older WHWTs.

\section{Factors associated} with owner acquisition of puppies under 8 weeks of age

\section{Rachel H Kinsman ${ }^{1}$, Rachel Casey ${ }^{1}$, Michelle Lord ${ }^{1}$, Joshua Woodward ${ }^{1}$, Séverine Tasker ${ }^{2,3}$, Toby Knowles ${ }^{2}$, Rosa Da Costa ${ }^{1}$, Jane Murray ${ }^{1}$}

1 Dogs Trust, London, United Kingdom

2 University of Bristol, Bristol, United Kingdom

3 Linnaeus Group, Shirley, United Kingdom

\section{OBJECTIVES}

To identify owner- and dog-related risk factors associated with acquisition of puppies under the recommended age of 8 weeks.

\section{METHODS}

The owner-reported data used in this analysis were collected from online questionnaires completed by puppy owners living in the UK and $\mathrm{ROI}$ who registered puppies $<16$ weeks of age into a longitudinal study.

Multivariable logistic regression was used to test for associations between acquisition at $<8$ weeks of age and potential explanatory variables (including breed (specific named/mixed/unknown), source of puppy, whether puppies were viewed with mother and/or father, previous dog ownership and number of times the puppy was viewed before acquisition).

\section{RESULTS}

Excluding home-bred puppies, 26.5\% (423/1597) were acquired $<8$ weeks of age. Two variables were retained in the model:

1. Owners who had owned other dogs during adulthood were more likely to acquire puppies $<8$ weeks old, compared with 'first time owners' or those 'who had a family dog as a child but not subsequently' (OR $=1.45,95 \% \mathrm{Cl} 1.09-1.92$, $P<0.010$ )

2. owners who visited their puppy at least once before acquisition were more likely to obtain puppies $<8$ weeks old, compared with owners who did not see their puppy before acquisition $(\mathrm{OR}=1.74,95 \% \mathrm{Cl}$ 1.34-2.27, $P<0.001)$

\section{STATEMENT (CONCLUSIONS)}

Advice regarding minimum acquisition age was not followed by $26.5 \%$ of this cohort, particularly those with 